\title{
Aleitamento materno exclusivo de prematuros e motivos para sua interrupção no primeiro mês pós-alta hospitalar
}

\author{
Exclusive breastfeeding of premature infants and reasons for \\ discontinuation in the first month after hospital discharge \\ La lactancia materna exclusiva de prematuros y motivos para su \\ interrupción en el primer mes después de la alta hospitalaria
}

\author{
Ana Paula Esmeraldo Lima \\ Thaíla Corrêa Castral ${ }^{b}$ \\ Luciana Pedrosa Leal ${ }^{a}$ \\ Marly Javorski ${ }^{a}$ \\ Gabriela Cunha Schechtman Sette ${ }^{a}$ \\ Carmen Gracinda Silvan Scochic \\ Maria Gorete Lucena de Vasconcelos ${ }^{a}$
}

\section{Como citar este artigo:}

Lima APE, Castral TC, Leal LP, Javorski M, Sette GCS, Scochi CGS, Vasconcelos MGL. Aleitamento materno exclusivo de prematuros e motivos para sua interrupção no primeiro mês pós-alta hospitalar. Rev Gaúcha Enferm. 2019;40:e20180406. doi: https://doi.org/10.1590/19831447.2019.20180406. a Universidade Federal de Pernambuco (UFPE), Departamento de Enfermagem. Recife, Pernambuco, Brasil.

• Universidade Federal de Goiás (UFG), Faculdade de Enfermagem e Nutrição, Departamento de Enfermagem. Goiânia, Goiás, Brasil.

'Universidade de São Paulo (USP), Escola de Enfermagem de Ribeirão Preto. Ribeirão Preto, São Paulo, Brasil.

\section{RESUMO}

Objetivo: Estimar a prevalência de aleitamento materno exclusivo de prematuros na alta hospitalar, aos 15 e 30 dias pós-alta, e identificar as alegações maternas para sua interrupção. Métodos: Estudo transversal com 108 prematuros nascidos em dois Hospitais Amigos da Criança, entre abril-julho de 2014. Realizou-se pesquisa em prontuário e entrevistas por telefone. Usaram-se análise descritiva, qui-quadrado de Pearson e teste exato de Fisher, com intervalo de confiança de $95 \%$.

Resultados: A prevalência do aleitamento materno exclusivo na alta foi de 85,2\%, de 75\% aos 15 dias e 46,3\% aos 30 dias. A principal alegação para introdução de outros alimentos e/ou líquidos foi o leite insuficiente.

Conclusões: Houve redução significativa nas taxas de aleitamento materno exclusivo após a alta, apontando a importância do acompanhamento pós-alta para reduzir o desmame precoce, sobretudo com ações educativas que previnam as insuficiências reais e percebidas na oferta de leite.

Palavras-chave: Aleitamento materno. Recém-nascido prematuro. Alta do paciente. Continuidade da assistência ao paciente. Desmame

\section{ABSTRACT}

Objective: To estimate the prevalence of exclusive breastfeeding premature infants at hospital discharge, and after 15 and 30 days, and to identify the mothers' claims for discontinuation.

Methods: Cross-sectional study with 108 premature infants born in two Baby-Friendly Hospitals between April and July 2014. A survey was conducted on medical records and through telephone interviews. A descriptive analysis, Pearson's chi-square test, and Fisher's exact test were used, with a 95\% confidence interval.

Results: The prevalence of exclusive breastfeeding at discharge was $85.2 \%, 75 \%$ after 15 days, and $46.3 \%$ after 30 days. The main claim for the introduction of other foods and/or liquids was insufficient milk.

Conclusions: There was a significant reduction in the rates of exclusive breastfeeding after discharge, pointing out the importance of continuity of patient care to reduce early weaning, especially with educational actions that help prevent real and perceived deficits in milk supply.

Keywords: Breastfeeding. Infant, premature. Patient discharge. Continuity of patient care. Weaning.

\section{RESUMEN}

Objetivo: Estimar la prevalencia de lactancia materna exclusiva de prematuros en el alta hospitalario, a los 15 y 30 días luego del alta, e identificar las alegaciones maternas para su interrupción.

Métodos: Estudio longitudinal con 108 prematuros nacidos en dos Hospitales Amigos del Niño, entre abril y julio de 2014. Se realizó una investigación en historiales y entrevistas por teléfono. Se utilizaron el análisis descriptivo, el chi-cuadrado de Pearson y el examen exacto de Fisher, con intervalo de confianza de $95 \%$.

Resultados: La prevalencia de la lactancia materna exclusiva luego del alta fue de $85,2 \%$, de $75 \%$ a los 15 días y de 46,3\% a los 30 días. La principal alegación para la introducción de otros alimentos y/o líquidos fue la insuficiente leche.

Conclusiones: Hubo una reducción significativa en las tasas de lactancia materna exclusiva después del alta, lo que señala la importancia del seguimiento post-alta para reducir el destete precoz, sobre todo con acciones educativas que ayuden a prevenir las insuficiencias reales y percibidas en la oferta de leche.

Palabras clave: Lactancia materna. Recién nacido prematuro. Alta del paciente. Continuidad de la atención al paciente. Destete. 


\section{—INTRODUÇÃO}

O Aleitamento Materno Exclusivo (AME) até o sexto mês de vida, recomendado pela Organização Mundial da Saúde (OMS), é o alimento ideal para a promoção do crescimento e desenvolvimento saudável. Além disso, favorece a sustentabilidade e a redução das desigualdades sociais, com potencial de contribuir para o cumprimento de vários Objetivos de Desenvolvimento Sustentável, a serem alcançados por todos os Estados-Membros das Nações Unidas até 2030 (1-2).

Para os Recém-Nascidos (RN) prematuros, o leite materno oferece benefícios adicionais, como menor incidência e gravidade de enterocolite necrosante, sepse e retinopatia da prematuridade, aumento no desempenho neuropsicomotor, fortalecimento do vínculo mãe-filho, menor tempo de hospitalização e menor incidência de reinternações ${ }^{(3)}$.

Entretanto, esses RN apresentam início tardio e menor duração do Aleitamento Materno (AM), quando comparados aos nascidos a termo ${ }^{(4-5)}$. Embora pesquisas tenham evidenciado melhoria na prática do AM entre prematuros, as taxas de AME nesse grupo estão inferiores às recomendadas pela OMS, que considera situação "boa" ou "muito boa" quando 50 a $89 \%$ e $90 \%$ a 100\%, respectivamente, das crianças menores de seis meses encontram-se em AME, independentemente da idade gestaciona|(6).

Mesmo quando se consegue estabelecer o AME em prematuros hospitalizados, muitos são desmamados em tempo inoportuno, já nas primeiras semanas após a alta hospitalar, com taxas decrescentes de AME que variam de $25 \%$ a $7,5 \%$ no primeiro mês pós-alta. Essas prevalências revelam a carência de intervenções de suporte materno e apoio à manutenção da amamentação, apesar dos benefícios incontestes que o leite humano proporciona a essas crianças ${ }^{(7-8)}$.

O planejamento e estabelecimento de intervenções efetivas que possibilitem a manutenção do AM do prematuro por tempo oportuno devem ser realizados com base no contexto epidemiológico, evidenciado por dados acerca da prática do AM no ambiente hospitalar, bem como sua realidade no período pós-alta e os motivos que conduzem as mães a introduzir outros alimentos. Nesse sentido, objetivou-se estimar a prevalência do AME de prematuros na alta hospitalar, aos 15 dias e 30 dias pós-alta, e identificar as alegações maternas para sua interrupção.

\section{MÉTODOS}

Pesquisa oriunda da tese "Aleitamento materno em prematuros hospitalizados e no primeiro mês pós-alta"(9). Trata-se de estudo transversal, desenvolvido em dois Hospitais Amigos da Criança, na cidade de Recife, Pernambuco, no período de abril a julho de 2014. Ambos são públicos, universitários, de referência regional quanto ao atendimento à gestação de alto risco, dispondo de Unidade de Terapia Intensiva Neonatal (UTIN) e banco de leite humano.

A população de referência foi constituída por prematuros nascidos nos referidos hospitais e suas respectivas mães. A amostra foi calculada por meio do programa Epitable no software Epi Info ${ }^{\mathrm{TM}}$ 7, considerando-se a população de 1363 prematuros nascidos no ano de 2012 em ambos hospitais, a prevalência de AME na alta hospitalar de $92 \%$ (estimada pelos primeiros vinte casos de cada instituição participante do estudo), poder de $80 \%$, erro amostral de $5 \%$ e nível de significância de 95\%, totalizando 105 prematuros. A esse total foram acrescidos 20\% para possíveis perdas.

Foram incluídos na amostra RN com menos de 37 semanas de idade gestacional, internados na unidade neonatal até as primeiras 48 horas de vida, com no mínimo 48 horas de permanência na unidade. Considerou-se unidade neonatal como a UTIN, a unidade de cuidados intermediários e a unidade canguru. Os critérios de exclusão compreenderam transferência do RN para outra instituição, óbito neonatal, situações de adoção/abandono de menor, presença de anormalidades congênita ou cromossômica que impossibilitavam o desenvolvimento da capacidade motora oral normal, bem como presença de galactosemia.

Considerou-se como critério de inclusão materno: ter filho internado na unidade neonatal, conforme critérios de inclusão do prematuro. Os critérios de exclusão compreenderam transferência ou óbito materno, uso de medicamentos incompatíveis com a amamentação, como antineoplásicos e radiofármacos, e presença de contraindicação temporária ou definitiva para amamentar, como infecção materna pelo Vírus da Imunodeficiência Humana (HIV) e pelo Vírus T-Linfotrópico Humano (HTLV) (2). Somente quando ambos, mãe e filho, atendiam a tais critérios, é que eram considerados elegíveis para a pesquisa.

Dos 202 prematuros e suas respectivas mães que tiveram alta hospitalar no período do estudo, 150 prematuros e 136 mães atenderam aos critérios de inclusão; e 22 foram excluídos. Como haviam 14 gemelares, a população de mães foi menor do que a de prematuros (Figura 1).

Optou-se por uma amostragem em sequência, que envolve recrutar todos os indivíduos de uma população acessível que atendam aos critérios de elegibilidade ao longo de um intervalo de tempo específico ${ }^{(10)}$. Houve perda de 20 binômios, todas pela impossibilidade de contato telefônico, após um mínimo de 15 tentativas, cujas ligações foram identificadas como fora da área de cobertura ou telefone desligado, ou número inexistente, sendo a amostra final composta por 108 prematuros e 94 mulheres (Figura 1). 


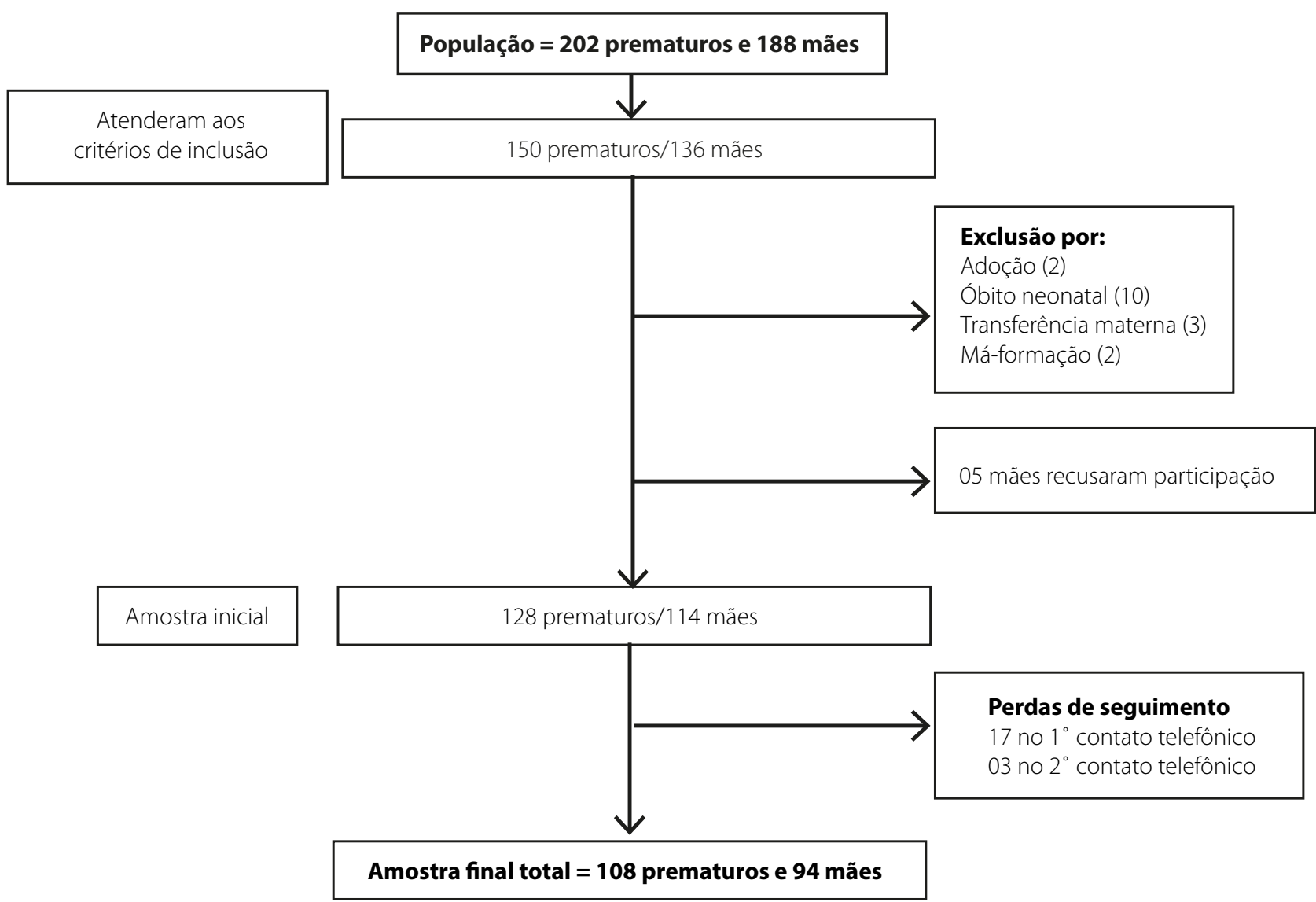

Figura 1 - Fluxograma do processo de obtenção da amostra do estudo. Recife, PE, Brasil, 2014 Fonte: Autores.

As visitas às unidades neonatais eram realizadas no mínimo três vezes por semana, pela pesquisadora principal e por uma auxiliar de pesquisa, para que as mães pudessem ser convidadas a participar do estudo e assinar o Termo de Consentimento Livre e Esclarecido, bem como para possibilitar o acesso aos prontuários tão logo ocorresse a alta hospitalar. Para facilitar o controle, foi elaborado um instrumento de seguimento dos participantes, executado desde a admissão do prematuro até o $30^{\circ}$ dia pós-alta hospitalar.

Para melhorar a acurácia do estudo, a auxiliar de pesquisa foi capacitada para a coleta dos dados no prontuário e era mantido contato telefônico semanal para discutir os procedimentos na coleta e resolver eventuais dificuldades. A etapa das entrevistas por telefone foi realizada exclusivamente pela pesquisadora principal.

Utilizou-se um formulário semiestruturado para a coleta de dados, construído e validado pelo grupo de pesquisa do projeto multicêntrico ao qual esta pesquisa está aninhada. O teste piloto foi realizado nas cinco regiões do país para adequação dos instrumentos e procedimentos de coleta de dados. Nesse estudo foram analisadas as variáveis relacionadas ao nascimento, internação e alimentação do prematuro.

No momento da alta hospitalar, eram coletados do prontuário os dados do nascimento e da internação do prematuro, incluindo idade gestacional, peso ao nascer, tempo de internação e tipo de aleitamento no momento da alta. Após a alta hospitalar, as mães foram entrevistadas por telefone aos 15 e 30 dias após a alta, obtendo-se informações quanto à situação do AM. Nos casos em que o RN não se encontrava em AME, solicitou-se que a mãe apresentasse o principal motivo para a introdução de outro alimento (incluindo água e chá) na dieta do prematuro, considerado como alegação materna para o não AME. Tais causas foram categorizadas em causas culturais/educacionais, sociais e biológicas. Esse questionamento não foi realizado para as mães que já não estavam em AME na avaliação anterior. As mães cujos filhos não se encontravam em AME na alta ou aos 15 dias continuaram a ser acompanhadas a fim de se avaliar o tipo de aleitamento. 
Para fins de categorização e análise, foram consideradas as definições propostas pela OMS ${ }^{(11)}$. Aleitamento Materno Exclusivo (AME): quando o leite materno é o único alimento, direto da mama ou ordenhado, ou leite humano de outra fonte, durante as últimas 24 horas; Aleitamento Materno Predominante (AMP): o leite materno é a fonte predominante de nutrição da criança, porém também recebe água, suco, chá, solução de sais de hidratação oral; Aleitamento Materno (AM): recebe leite materno e leite não humano durante as últimas 24 horas; Alimentação de Substituição (AS), quando a criança não recebe leite humano, sendo alimentada somente com substitutos do leite materno.

A partir dos instrumentos preenchidos manualmente, procedeu-se à dupla entrada dos dados, para verificação de possíveis inconsistências e erros de digitação. O processamento e análise dos dados foi realizado utilizando-se o programa Statistical Package for the Social Sciences (SPSS, versão 21.0). Para avaliação das variáveis categóricas, foram calculadas as frequências percentuais e construídas as respectivas distribuições de frequência; para as variáveis contínuas, foram calculadas as medidas de tendência central (média e desvio padrão). As prevalências dos tipos de AM foram estimadas para cada momento de coleta: alta hospitalar, quinze e trinta dias após a alta. Para a comparação das prevalências de AME, bem como das causas alegadas para sua interrupção, nos períodos avaliados, utilizou-se o teste de diferença de proporções (Teste Exato de Fisher ou Qui-quadrado de Pearson), com intervalo de confiança de 95\% (IC95\%).

Atendendo à Resolução 466/2012 do Conselho Nacional de Saúde, a pesquisa foi aprovada pelo Comitê de Ética em Pesquisa do Centro Integrado de Saúde Amaury de Medeiros, da Universidade de Pernambuco (Parecer $n^{\circ}$ 631.464, de 24/04/2014). Todas as participantes assinaram o Termo de Consentimento Livre e Esclarecido. O estudo integra o projeto multicêntrico "Aleitamento materno em prematuros: impacto da IHAC para unidades neonatais".

\section{RESULTADOS}

Dos 108 prematuros participantes, 63 (58,3\%) eram do sexo feminino, 14 (13,0\%) gemelares e 91 (84,3\%) eram prematuros moderados a tardios, com idade gestacional média de $33,7( \pm 2,2)$ semanas. A maioria apresentava peso de nascimento entre 1500 gramas e 2499 gramas $(65,1 \%)$, com uma média de peso de 1964 ( \pm 533) gramas. O tempo médio de internação foi de 21 dias (3-94 dias) (Tabela 1).
Tabela 1 - Características dos prematuros segundo variáveis do nascimento e internação. Recife, PE, Brasil, 2014

Variáveis

$\mathbf{N}$ $\%$

Sexo

Feminino

$63 \quad 58,3$

Masculino

45

41,7

\section{Gemelar}

$\operatorname{Sim}$

$14 \quad 13,0$

Não

$94 \quad 87,0$

\section{Idade gestacional (semanas)}

Menor de 28 semanas

$4 \quad 3,7$

De 28 a 31 semanas

$13 \quad 12,0$

De 32 a 36 semanas

$91 \quad 84,3$

$$
\begin{aligned}
& \text { Mínimo - Máximo } \\
& \text { Média ( } \pm \text { Desvio padrão) }
\end{aligned}
$$$$
27,0-36,9
$$$$
33,7( \pm 2,2)
$$

\begin{tabular}{|c|c|}
\hline Menos de $1500 \mathrm{~g}$ & 21 \\
\hline $1500 \mathrm{~g}$ a $2499 \mathrm{~g}$ & 69 \\
\hline $2500 \mathrm{~g}$ ou mais & 16 \\
\hline Mínimo - Máximo & 620 \\
\hline Média ( \pm Desvio padrão) & 196 \\
\hline
\end{tabular}

\section{Peso ao nascer (gramas) ${ }^{(1)}$}

\section{Tempo de internamento (dias)}

$\begin{array}{lc}\text { Mínimo - Máximo } & 3-94 \\ \text { Média ( } \pm \text { Desvio padrão) } & 21( \pm 19)\end{array}$

Fonte: Dados da pesquisa, 2014

(1) 0 total não soma o valor de N, por inconsistência no prontuário.

O tipo de aleitamento que predominou no momento da alta, após 15 dias e 30 dias da alta hospitalar foi o AME, porém esta prevalência sofreu uma redução entre estes períodos. A prevalência de AME na alta foi de $85,2 \%$, caindo para $75 \%$ e $46,3 \%$ aos 15 e 30 dias após a alta, respectivamente. Houve um declínio na proporção dos prematuros que estavam em AME de 54,3\%, da alta até o final do primeiro mês pós-alta, e o teste de homogeneidade foi altamente significativo ( $p$-valor $<0,001$ ), indicando que essa redução foi relevante entre os três períodos avaliados (Tabela 2). 
Tabela 2 - Tipo de aleitamento materno nos três períodos avaliados. Recife, PE, Brasil, 2014

\begin{tabular}{|c|c|c|c|c|}
\hline \multirow{2}{*}{ Variáveis } & \multicolumn{3}{|c|}{ Período da avaliação } & \multirow{2}{*}{$p$-valor } \\
\hline & Alta & 15 dias & 30 dias & \\
\hline \multicolumn{4}{|l|}{ AME } & \multirow{3}{*}{$<0,001^{(5}$} \\
\hline Sim & $92(85,2 \%)$ & $81(75,0 \%)$ & $50(46,3 \%)$ & \\
\hline Não & $16(14,8 \%)$ & $27(25,0 \%)$ & $58(53,7 \%)$ & \\
\hline \multicolumn{4}{|l|}{ Tipo de AM } & \\
\hline $\operatorname{AME}^{(1)}$ & $92(85,2 \%)$ & $81(75,0 \%)$ & $50(46,3 \%)$ & \multirow{4}{*}{$<0,001^{(6}$} \\
\hline $\mathrm{AMP}^{(2)}$ & $0(0,0 \%)$ & $8(7,4 \%)$ & $10(9,3 \%)$ & \\
\hline $\mathrm{AM}^{(3)}$ & $14(13,0 \%)$ & $17(15,7 \%)$ & $39(36,1 \%)$ & \\
\hline $\mathrm{AS}^{(4)}$ & $2(1,8 \%)$ & $2(1,9 \%)$ & $9(8,3 \%)$ & \\
\hline
\end{tabular}

Fonte: Dados da pesquisa, 2014

${ }^{(1)}$ AME: Aleitamento materno exclusivo. ${ }^{(2)}$ AMP: Aleitamento materno predominante. ${ }^{(3)}$ AM: Aleitamento materno. ${ }^{(4)}$ AS: Alimentação de substituição. ${ }^{(5)} p$-valor do teste Qui-quadrado de Pearson. ${ }^{(6)} p$-valor do teste Exato de Fisher.

As alegações maternas para a introdução de outros alimentos, que não o leite materno, aos 15 e aos 30 dias após a alta hospitalar encontram-se descritos na Tabela 3. Verificou-se que aos 15 dias os motivos alegados mais frequentemente pelas mães foram: leite insuficiente/secou (31,3\%), crença no benefício do chá $(25,0 \%)$ e necessidade de água (18,8\%). Aos 30 dias, os motivos mais citados para a interrupção do AME foram: leite insuficiente/secou (77,5\%) e necessidade de água (9,7\%). O teste de homogeneidade não foi significativo na comparação das alegações nos dois momentos avaliados ( $p$-valor $=0,312$ ), indicando que os motivos para a não realização do AME nos dois momentos foram semelhantes.

Tabela 3 - Alegações maternas para oferta de outros líquidos/alimentos aos 15 dias e aos 30 dias pós-alta hospitalar. Recife, PE, Brasil, 2014

\section{Motivos avaliados}

\section{Causas culturais/educacionais}

Crença no benefício do chá

Necessidade de água

Leite insuficiente/secou

\section{Causas sociais}

Orientação profissional de saúde

Conveniência materna

Influência de terceiros

\section{Causas biológicas}

Bebê não suga/sonolento

Bebê não dorme/chora muito

Total

Fonte: Dados da pesquisa, 2014

(1) $p$-valor do teste Qui-quadrado de Pearson

\begin{tabular}{|c|c|c|c|}
\hline 15 dias & & & \\
\hline$\%$ & $\mathbf{N}$ & $\%$ & \\
\hline
\end{tabular}

$4 \quad 25,0=12=3,2$

$3 \quad 18,8$

$3 \quad 9,7$

$\begin{array}{llll}5 & 31,3 & 24 & 77,5\end{array}$

$\begin{array}{lllll}1 & 6,2 & 1 & 3,2 & 0,312^{(1)} \\ 0 & 0,0 & 1 & 3,2 & \\ 0 & 0,0 & 1 & 3,2 & \end{array}$

\begin{tabular}{lccc}
2 & 12,5 & 0 & 0,0 \\
1 & 6,2 & 0 & 0,0 \\
16 & 100 & 31 & 100 \\
\hline
\end{tabular}




\section{DISCUSSÃO}

As prevalências de AME encontradas neste estudo apresentaram valores expressivos quando comparadas às taxas verificadas em outras regiões do país e do mundo. A prevalência do AME de prematuros no momento da alta hospitalar mostrou-se superior à de pesquisa transversal realizada em um Hospital Amigo da Criança do sudeste do Brasil, na qual apenas $47,6 \%$ dos prematuros encontravam-se em AME no momento da alta ${ }^{(12)}$. Em coorte realizada na mesma região, com prematuros $<33$ semanas, essa taxa foi de apenas 5,5\% no momento da alta ${ }^{(7)}$. No entanto, evidências indicam que menores de 32 semanas têm maior risco de desmame precoce ${ }^{(5)}$.

Estudo multicêntrico longitudinal, de base populacional, realizado em oito cidades de diferentes países, incluindo o Brasil, também encontrou taxa inferior à dessa pesquisa. Avaliou-se os padrões de crescimento de prematuros $<37$ semanas de idade gestacional e suas práticas de alimentação do nascimento aos 6 meses de vida, evidenciando que $72,0 \%$ dos prematuros recebiam alta hospitalar em AME ${ }^{(13)}$. O mesmo ocorreu em estudo prospectivo com 1488 prematuros $<37$ semanas de idade gestacional, que identificou uma taxa de 68,0\% de AME no mesmo período. Tal estudo foi realizado na Dinamarca, país desenvolvido, onde as mulheres têm, no mínimo, 10 meses de licença maternidade remunerada ${ }^{(14)}$.

Esses resultados mostraram que é possível estabelecer - AME na maioria dos prematuros hospitalizados, apesar de suas peculiaridades, que desafiam o início e o estabelecimento da amamentação. O apoio de profissionais de saúde capacitados e realização de uma prática centrada nas necessidades da criança e da família, são fundamentais para promover o AM.

Terem nascido em Hospitais Amigos da Criança possivelmente pode ter contribuído para esse resultado, considerando que um dos objetivos dessa Iniciativa é a meIhoria dos índices de AM, em especial o exclusivo, através da implementação dos Dez Passos, mesmo essa estratégia tendo como foco o RN a termo.

De acordo com a II Pesquisa Nacional de Prevalência de Aleitamento Materno nas Capitais Brasileiras e no Distrito Federal, a duração média do AME entre crianças que nasceram em Hospitais Amigo da Criança foi de 60,2 dias, em comparação aos 48,1 dias entre crianças que não nasceram em Hospitais Amigo da Criança. Bebês nascidos em Hospitais Amigo da Criança também aumentaram a chance em 6\% para a amamentação no primeiro dia em casa após a alta da maternidade; em 13\% para o AME em menores de dois meses, 8\% para o AME em menores de três meses e 6\% para o AME em menores de seis meses ${ }^{(6)}$.

Contudo, ao observar a evolução da prevalência do AME desses prematuros no período após a alta hospitalar, observou-se uma queda acentuada no primeiro mês pós-alta. Dados semelhantes foram obtidos em pesquisa no sul do país, que identificou uma prevalência de AME em prematuros aos 15 e 45 dias após a alta de 71,5\% e 45,2\%, respectivamente ${ }^{(15)}$. Por sua vez, uma coorte prospectiva com prematuros de idade gestacional de 33 a 36 semanas, realizado em Hospital Amigo da Criança, também no sul do Brasil, evidenciou menores taxas de AME, de 36,2\% no $14^{\circ}$ dia após a alta e de $25 \%$ no $28^{\circ}$ dia ${ }^{(8)}$.

Os primeiros trinta dias após a alta hospitalar são considerados críticos para a adaptação da mãe-prematuro-família, tornando-se essencial não apenas uma equipe de saúde neonatal comprometida e qualificada no âmbito hospitalar, mas que também coexista no nível de atenção básica profissionais capacitados a atenderem os prematuros e sua família em suas necessidades, incluindo a promoção do AM, possibilitando a continuidade da assistência, com articulação das ações entre os diferentes níveis de atenção à saúde, eixo principal das redes de atenção à saúde.

Em ambos os hospitais participantes do estudo, havia Banco de Leite Humano, era permitido o livre acesso aos pais na UTIN e eram realizadas orientações quanto à importância do leite materno, especialmente para o prematuro, apoio para manter a lactação com ordenhas frequentes, oferta do leite materno cru ou pasteurizado e preparo do prematuro e sua família para a alta hospitalar. Ainda que essas práticas sejam recomendadas na literatura ${ }^{(5)}$, parecem não ser suficientes para manutenção das taxas de AME verificadas no momento da alta. A queda significativa do AME, logo nas primeiras semanas pós-alta, revela as dificuldades do binômio durante sua adaptação à rotina domiciliar.

As alegações maternas para a oferta de outros líquidos ou alimentos foram principalmente causas de ordem cultural/educacional, como o fato do leite ser insuficiente ou ter secado, ou da crença do benefício do chá e da necessidade da oferta de água. Essas alegações foram semelhantes no $15^{\circ}$ ou no $30^{\circ}$ dia pós-alta.

Esses achados são consistentes com outros estudos da literatura. Em pesquisa de coorte com RN a termo ou pré-termo, identificaram-se como principais causas alegadas para o desmame nos primeiros 15 dias de vida a redução do volume de leite e o leite fraco ${ }^{(16)}$. A quantidade insuficiente de leite também foi evidenciada como a principal causa alegada para a interrupção do AM de prematuros em estudo que comparou duas coortes contemporâneas 
na Austrália Ocidental ${ }^{(17)}$. Por sua vez, estudo italiano verificou que o relato materno de dificuldade em fornecer uma quantidade adequada de leite ao filho esteve significativamente associado a um maior risco de desmame ${ }^{(18)}$.

Vale destacar que a percepção materna de pouco leite é também uma das razões mais comuns alegadas pelas mães de bebês nascidos a termo ${ }^{(19)}$. Na presente pesquisa, as justificativas por causa biológica, quando o bebê não sugava ou era sonolento, peculiaridade do prematuro reconhecida como dificultadora do AM, foi referida apenas por pequena parcela das mães, fato que pode ser justificado pela maturidade do RN no momento da alta, já que a média da idade corrigida nesse período foi de 37 semanas.

A influência do fator cultural, portanto, deve ser sempre levado em consideração nas práticas de orientação e promoção do AM, no sentido de empoderar a mulher na sua capacidade de aleitar o filho, mesmo prematuro.

O relato de pouco leite é um problema complexo, que transpõe linhas culturais, geográficas e socioeconômicas. É sabido que a percepção da produção insuficiente de leite ocorre muito mais frequentemente do que o problema real. Leite insuficiente, real ou percebido, é descrito como uma mãe sentindo que sua produção de leite é insuficiente tanto para satisfazer a fome do seu filho, quanto para proporcionar um ganho ponderal adequado. No entanto, poucas mulheres têm problemas genuínos com a sua produção láctea, sendo as causas secundárias muito mais comuns ${ }^{(20)}$.

Cabe assinalar que os resultados obtidos se referem a um período de relativa vulnerabilidade, onde mãe e família estão em fase de adaptação com a chegada do prematuro no domicílio, sendo frequente momentos de estresse, ansiedade e cansaço materno. É sabido que a ansiedade materna e o nascimento prematuro afetam negativamente a lactogênese, levando a uma redução potencial no suprimento de leite materno ${ }^{(18)}$. Quando não há um suporte efetivo e adequado da rede de apoio, a produção láctea pode ser prejudicada.

O desmame parcial ou total também foi justificado pelas mães por causas de ordem social, sobretudo por falta de orientação adequada de profissional de saúde, conforme já apontado na literatura ${ }^{(16)}$. O suporte profissional deve ser consistente para conseguir influir positivamente a muIher nos seus esforços de amamentar um bebê prematuro.

A manutenção do AME após a alta hospitalar deve ser um objetivo comum para os profissionais que assistem o binômio mãe-bebê e família. O ambiente da unidade neonatal frequentemente fornece o suporte necessário às mães, mas é em casa que elas se deparam com dúvidas e dificuldades, associadas muitas vezes a apoio profissional e social insuficiente, dificultando a continuidade do AME.

\section{CONCLUSÕES}

Os resultados encontrados evidenciam que é possível atingir boas taxas de AME na alta hospitalar dos prematuros. No entanto, a redução significativa na prevalência do AME ao longo do primeiro mês no domićlio demonstra que o acompanhamento nessa fase é fundamental para se evitar o desmame precoce.

É preciso que haja um planejamento de alta, que envolva mãe-família e equipe multidisciplinar, e uma rede de atenção à saúde eficiente, com ações articuladas entre a unidade hospitalar de referência e o serviço de atenção básica que garantam o cuidado integral por meio da oferta de serviços contínuos nos diferentes níveis de complexidade.

Considerando-se que os principais motivos relatados pelas mães para o desmame total ou parcial foram de ordem educacional/cultural, faz-se necessário, sobretudo, acompanhamento e orientações quanto ao processo de amamentação e produção láctea, ajudando a prevenir as insuficiências reais e percebidas na oferta de leite.

Esses resultados podem auxiliar no planejamento de estratégias voltadas ao ensino, pesquisa e assistência em prol do AM nesse segmento populacional de alto risco, englobando o cuidado à mulher, criança e família, nos diversos cenários dos serviços de saúde.

É necessário que novos estudos sejam realizados, com outros delineamentos, para melhor compreensão do fenômeno, que transcende aspectos quantitativos, e que novas intervenções educativas de promoção, proteção e apoio ao aleitamento materno, sobretudo na atenção básica de saúde, que envolvam as peculiaridades dos prematuros, possam ser testadas e implementadas, com vistas a aumentar a duração do AME e, consequentemente, contribuir com a melhora da saúde dessas crianças.

Como limitação do estudo, menciona-se o método adotado para coleta de dados, por telefone, sujeito a perdas de contato, o que foi minimizado pela obtenção, sempre que possível, de mais de um número de telefone, além de realizar várias tentativas de discagem, em diferentes horários.

\section{口 REFERÊNCIAS}

1. Sartorio BT, Coca KP, Marcacine KO, Abuchaim EDSV, Abrão ACFDV. Breastfeeding assessment instruments and their use in clinical practice. Rev Gaúcha Enferm. 2017;38(1):e64675. doi: https://doi.org/10.1590/1983-1447.2017.01.64675.

2. Ministério da Saúde (BR). Secretaria de Atenção à Saúde. Departamento de Atenção Básica. Saúde da criança: aleitamento materno e alimentação complementar. 2. ed. Brasília (DF): Ministério da Saúde; 2015 [citado 2018 out 18]. Disponível em: http://bvsms.saude.gov.br/bvs/publicacoes/saude_ crianca_aleitamento_materno_cab23.pdf.

3. Quigley M, Embleton ND, McGuire W. Formula versus donor breast milk for 
feeding preterm or low birth weight infants. Cochrane Database Syst Rev. 2018;(6):CD002971. doi: https://doi.org/10.1002/14651858.CD002971.pub4.

4. Dereddy N, Talati A, Smith A, Kudumula R, Dhanireddy R. A multipronged approach is associated with improved breast milk feeding rates in very low birth weight infants of an inner-city hospital. J Hum Lact. 2015;31(1):43-6. doi: https://doi.org/10.1177/0890334414554619.

5. Freitas BAC, Lima LM, Carlos CFLV, Priore SE, Franceschini SCC. Duração do aleitamento materno em prematuros acompanhados em serviço de referência secundário. Rev Paul Pediatr. 2016;34(2):189-96. doi:https://doi.org/10.1016/j. rpped.2015.10.005.

6. Ministeŕrio da Saúde (BR). Secretaria de Atenção à Saúde. Departamento de Ações Programadas e Estratégicas. II Pesquisa de Prevalência de Aleitamento Materno nas Capitais Brasileiras e Distrito Federal. Brasilia (DF): Ministério da Saúde; 2009 [citado 2018 out 18]. Disponível em: http://bvsms.saude.gov.br/ bus/publicacoes/pesquisa_prevalencia_aleitamento_materno.pdf.

7. Méio MDBB, Villela LD, Júnior G, Tovar CM, Moreira MEL. Breastfeeding of preterm newborn infants following hospital discharge: follow-up during the first year of life. Cienc Saude Coletiva. 2018;23(7):2403-12. doi: https://doi. org/10.1590/1413-81232018237.15742016.

8. Azevedo M, Cunha MLC. Fatores associados ao aleitamento materno exclusivo em prematuros no primeiro mês após a alta hospitalar. Clin Biomed Res. 2013 [citado 2017 dez 10];33(1):40-9. Disponível em: http://seer.ufrgs.br/index. php/hcpa/article/view/37653/25670.

9. Lima APE. Aleitamento materno em prematuros hospitalizados e no primeiro mês pós-alta [tese]. Recife (PE): Universidade Federal de Pernambuco; 2016 [citado 2018 out 18]. Disponivel em: https://repositorio.ufpe.br/ handle/123456789/18037.

10. Polit DF, Beck CT. Essentials of nursing research: appraising evidence for nursing practice. 9th ed. Philadelphia: Wolters Kluwer Health; 2017.

11. World Health Organization (CH), United Nations Children's Fund. Guideline: updates on HIV and infant feeding: the duration of breastfeeding, and support from health services to improve feeding practices among mothers living with HIV. Geneva: WHO; 2016 [cited 2018 Oct 20]. Available from: https:// apps.who.int/iris/bitstream/handle/10665/246260/9789241549707-eng. pdf? sequence $=1$ \&isAllowed $=\mathrm{y}$.

12. Gomes ALM, Balaminut T, Lopez SB, Pontes KAES, Scochi CGS, Christoffel MM. Aleitamento materno de prematuros em hospital amigo da criança: da alta hospitalar ao domicílio. Rev RENE. 2017;18(6):810-7. doi: https://doi. org/10.15253/2175-6783.2017000600015.
13. Ismail LC, Giuliani F, Bhat BA, Bishop D, Papageorghiou AT, Ochieng R, et al. Preterm feeding recommendations are achievable in large-scale research studies. BMC Nutrition. 2016;2:9. doi: https://doi.org/10.1186/s40795-0160047-9.

14. Maastrup R, Hansen BM, Kronborg H, Bojesen SN, Hallum K, Frandsen A, et al. Factors associated with exclusive breastfeeding of preterm infants: results from a prospective national cohort study. PloS One. 2014;9(2):e89077. doi: https:// doi.org/10.1371/journal.pone.0089077.

15. Sassá AH, Schmidt KT, Rodrigues BC, Ichisato SMT, Higarashi IH, Marcon SS. Bebês pré-termo: aleitamento materno e evolução ponderal. Rev Bras Enferm. 2014 [citado 2018 jun 10];67(4):594-600. Disponível em: http://www.scielo. br/pdf/reben/v67n4/0034-7167-reben-67-04-0594.pdf.

16. Rocci E, Fernandes RAQ. Dificuldades no aleitamento materno e influência no desmame precoce. Rev Bras Enferm. [Internet]. 2014 [citado 2017 dez 04];67(1):22-7. Disponível em: http://www.redalyc.org/ pdf/2670/267030130003.pdf.

17. Sharp M, Campbell C, Chiffings D, Simmer K, French N. Improvement in longterm breastfeeding for very preterm infants. Breastfeed Med. 2015;10(3):1459. doi: http://online.liebertpub.com/doi/pdf/ https://doi.org/10.1089/ bfm.2014.0117.

18. Gianni ML, Bezze EN, Sannino P, Baro M, Roggero P, Muscolo S, et al. Maternal views on facilitators of and barriers to breastfeeding preterm infants. BMC Pediatr. 2018;18:283. doi: https://doi.org/10.1186/s12887-018-1260-2.

19. Oliveira CS, locca FA, Carrijo MLR, Garcia RDATM. Breastfeeding and complications that contribute to early weaning. Rev Gaúcha Enferm. 2015;36(esp):16-23. doi: https://doi.org/10.1590/1983-1447.2015.esp.56766.

20. Bergmann RL, Bergmann KE, Von Weizsäcker K, Berns M, Henrich W, Dudenhausen JW. Breastfeeding is natural but not always easy: intervention for common medical problems of breastfeeding mothers - a review of the scientific evidence. J Perinat Med. 2014;42(1):9-18. doi: https://doi.org/10.1515/jpm2013-0095.

\section{AGRADECIMENTOS}

Nosso agradecimento ao apoio e financiamento fornecido pela Fundação Bill e Melinda Gates (OPP1107597), Conselho Nacional de Desenvolvimento Cientíico e Tecnológico (CNPq) e Ministério da Saúde/Decit (processo n.401628/2013-2), a0 projeto multicêntrico "Aleitamento materno em prematuros: impacto da IHAC para unidades neonatais", ao qual esta pesquisa se vincula.

\section{- Autor correspondente:}

Ana Paula Esmeraldo Lima

Recebido: 13.11.2018

E-mail: anapaulaesmeraldo@gmail.com 